\title{
Reversible oxygen migration and phase transitions in hafnia-based ferroelectric devices
}

\author{
Pavan Nukala ${ }^{1,2 *}$, Majid Ahmadi ${ }^{1,3}$, Yingfen Wei1 ${ }^{1,3}$, Sytze de Graaf', Evgenios Stylianidis ${ }^{1,4}$, Tuhin Chakrabortty ${ }^{2}$, \\ Sylvia Matzen ${ }^{5}$, Henny W. Zandbergen ${ }^{6}$, Alexander Björling7, Dan Mannix ${ }^{8,9,10}$, Dina Carbone ${ }^{7}$, Bart Kooi ${ }^{1,3}$, \\ Beatriz Noheda $\mathbf{a}^{1,3 *}$ \\ 1'Zernike Institute of Advanced Materials, University of Groningen, 9747 AG Groningen, Netherlands. ${ }^{2}$ Centre for Nano Science and Engineering, Indian Institute of Science, \\ Bengaluru, 560012, India. ${ }^{3}$ CogniGron (Groningen Cognitive Systems and Materials Center), University of Groningen, 9747 AG Groningen, Netherlands. ${ }^{4}$ Department of \\ Physics and Astronomy, University College London, London WC1E 6BT, UK. ${ }^{5}$ Center for Nanoscience and Nanotechnology, Paris-Saclay University, CNRS, 91120 Palaiseau, \\ France. ${ }^{6}$ Kavli Institute of Nanoscience, Faculty of Applied Sciences, Delft University of Technology, 2628 CJ Delft, Netherlands. ${ }^{7}$ MAX IV Laboratory, Lund University, SE-221 \\ 00 Lund, Sweden. ${ }^{8}$ University Grenoble Alpes, CNRS, Institut Néel, 38042 Grenoble, France. ${ }^{9}$ European Spallation Source, SE-221 00 Lund, Sweden. ${ }^{10}$ Department of \\ Chemistry, Aarhus University, DK-8000 Aarhus, Denmark. \\ *Corresponding author. Email: pnukala@iisc.ac.in (P.N.); b.noheda@rug.nl (B.N.)
}

Unconventional ferroelectricity exhibited by hafnia-based thin films, robust at nanoscale sizes, presents tremendous opportunities in nanoelectronics. However, the exact nature of polarization switching remains controversial. We investigated $\mathrm{La}_{0.67} \mathrm{Sr}_{0.33} \mathrm{MnO}_{3} / \mathrm{Hf}_{0.5} \mathrm{Zr}_{0.5} \mathrm{O}_{2}$ capacitor interfaced with various top electrodes while in situ electrical biasing using atomic resolution microscopy with direct oxygen imaging, as well as synchrotron nanobeam diffraction. When the top electrode is oxygen reactive, we clearly show reversible oxygen vacancy migration with electrodes being the source and sink of oxygen, and the dielectric layer acting as a fast conduit at millisecond timescales. With non-reactive top electrodes and at longer time scales (seconds), the dielectric layer also acts as an oxygen source/sink. Our results show that ferroelectricity in hafnia-based thin films is unmistakably intertwined to oxygen voltammetry.

The discovery of silicon compatible nano-ferroelectricity in hafnia-based thin films (1) has triggered a vast amount of fundamental research and rejuvenated interest in ferroelectric materials in microelectronics, for low-power non-volatile memory and logic devices. This ferroelectricity is robust even at film thicknesses as low as $1 \mathrm{~nm}(2-4)$, a situation that was believed impossible based on the classical understanding of ferroelectricity. The spontaneous polarization observed in these films is ascribed generally to a metastable polar orthorhombic phase ( $P$ ca2 ${ }_{1}$, o-phase) (5). A higher energy rhombohedral phase $(R 3 m / R 3$, r-phase) has been reported for epitaxial growth of $\mathrm{Hf}_{0.5} \mathrm{Zr}_{0.5} \mathrm{O}_{2}$ (HZO) on $\mathrm{La}_{0.67} \mathrm{Sr}_{0.33} \mathrm{MnO}_{3}$ (LSMO) buffered perovskite and on trigonal substrates (6). Remnant polarization $\left(\mathrm{P}_{r}\right)$ values as high as $35 \mu \mathrm{C} / \mathrm{cm}^{2}$ were measured (7) on HZO. The pronounced effects of particle size reduction, surface effects, dopants, oxygen vacancies $\left(\ddot{V}_{o}\right)$, epitaxial strain and residual stresses at nanoscale have been investigated as possible reasons to stabilize these otherwise metastable phases in thin films $(5,8,9)$. On the other end of the size range, films as thick as $1 \mu \mathrm{m}$ (10) and bulk samples (11) are ferroelectric, with the stabilization due to dopant and defect chemistry $(10,12)$.

Armed with an understanding of the virgin state polarization, vibrant research is being conducted on the mechanism of polarization switching. The dynamics of this process, through the lens of a nucleation-limited switching model and pointing out the negligible role of domain growth (or domain wall motion), have been studied for doped hafnia films grown in the o-phase (13-19). Flat phonon bands and localized dipoles in half unit cells in the o-phase have been postulated as an intrinsic reason for switching without forming domain walls in these systems (20).

Hafnia-based and zirconia-based materials are also an important class of resistive memory devices and oxygen conductors that exhibit memristive hysteresis driven by $\ddot{\mathrm{V}}_{\text {o }}$ conduction and redox reactions $(21,22)$. More generally, in devices of thin-film ferroelectric oxides such as tunnel junctions, both $\ddot{V}_{0}$ migration and polarization switching lead to hysteresis (23-28). Understanding whether these effects are synergetic or independent is crucial to achieve device control (29-33). In tunnel junctions of HZO thin-films on LSMO buffered $\mathrm{SrTiO}_{3}$ (STO), Wei et al. (23) observed a divergence of the tunnel electro-resistance (TER), from $100 \%$ to $10^{6 \%}$ upon device cycling, which the authors explained as a possible transition from polarization switching to $\ddot{V}_{o}$ migration assisted switching. This observation suggested the two mechanisms are independent. Sulzbach et al. (24) also reported a similar divergence in the TER as a function of the applied 
voltage in HZO layers before breakdown. However, the electric polarization in hafnia has been theoretically proposed to originate from oxygen vacancies through electrostrictive effects, strongly suggesting the extrinsic nature of the polarization switching (29). Direct structural observations during polarization switching can potentially resolve these controversies (34).

We report operando atomic scale electron microscopy investigations of LSMO/HZO/LSMO capacitor stacks grown on conducting (Nb-doped) STO substrate under electric field (35). LSMO is a standard choice of bottom electrode in complex oxide devices $(23-26,31)$ and so our conclusions are relevant for understanding a wider class of devices. We performed in situ biasing measurements while employing two scanning transmission electron microscopy (STEM) imaging modes, high-angle annular dark-field (HAADF) STEM and integrated differential phase contrast (iDPC) STEM. With iDPC STEM, we recently imaged hydrogen atoms next to $\mathrm{Ti}$ metal atoms (36), demonstrating this robust atomic resolution imaging technique for simultaneously measuring heavy and light elements. By directly imaging oxygen, we provide evidence of the reversible and hysteretic migration of $\ddot{\mathrm{V}}_{\mathrm{o}}$ from the bottom to the top electrode through the HZO layer. Associated with such migration, we show $\ddot{\mathrm{V}}_{\mathrm{o}}$ induced phase transitions in LSMO (bottom electrode) and HZO layers. Additionally, through operando x-ray diffraction, ex situ microscopy, and transport measurements on devices with both noble $(\mathrm{Au})$ and oxygen reactive top electrodes (LSMO, TiN), we show that oxygen voltammetry is also found at short timescales (millisecond and less). We believe these observations clearly show that polarization switching and oxygen voltammetry are not independent. The long-term effects include redox-based and topotactic phase transitions in both HZO and LSMO layers.

We begin by showing the evolution of the epitaxial LSMO layer (bottom electrode) with bias, with the voltage applied to the top electrode, and keeping the bottom electrode at $0 \mathrm{~V}$ (fig. S1) (22). Our iDPC-STEM image of the virgin state (Fig. $1 \mathrm{~A}$ and fig. S2A) shows the antiphase octahedral $\delta$ tilts present in the LSMO perovskite structure (22). Mn-O-Mn bond angles, as we measured in various s regions, are between $165^{\circ}-176^{\circ}$ (37). Upon increasing the bias to $2 \mathrm{~V}$, a noticeable displacement of Mn columns away from the center of oxygen octahedra (Fig. 1B) appears throughout the film (barring the first three monolayers at the interface with $\mathrm{Nb}$ :STO). These displacements are randomly oriented with a mean value of $18.6 \mathrm{pm}$ and std. deviation of $10.2 \mathrm{pm}$ (fig. S3), indicating a transformation from an $\mathrm{MnO}_{6}$ octahedral toward an $\mathrm{MnO}_{5}$ square pyramidal coordination (35). Thus, at $2 \mathrm{~V}$ the LSMO film contains a combination of $\mathrm{MnO}_{5}$ and $\mathrm{MnO}_{6}$ polyhedra. While this structural feature was not previously observed for LSMO, Brownmillerite (BM, oxygen deficient perovskite) phases are reported to exhibit $\mathrm{MnO}_{5}$ square pyramids in the parent compound, $\mathrm{SrMnO}_{3}$ (38). We refer to this $\mathrm{MnO}_{5}$ $\mathrm{MnO}_{6}$ combination as a BM-precursor phase (39). As for the first 3 monolayers, an important feature is the exaggerated antiphase $\delta$ tilts, with $\mathrm{Mn}-\mathrm{O}-\mathrm{Mn}$ bond angles of $143-146^{\circ}$ that are not typical of perovskite structures (fig. S2, B to D).

Upon increasing the biasing voltage to $4 \mathrm{~V}$, LSMO converts into a well-studied BM phase $(40,41)$ except for the first few monolayers near the interface with the substrate, which transform to the BM-precursor phase (fig. S4A). Transformation from perovskite to $\mathrm{BM}$ phase occurs via $\ddot{\mathrm{V}}_{\mathrm{o}}$ ordering in every alternate $\mathrm{Mn}-\mathrm{O}$ plane along the $c$ '-axis (Fig. 1C), transforming the Mn coordination from octahedral or square pyramidal to tetrahedral. Back-to-back $\mathrm{MnO}_{4}$ tetrahedra along [1-10] alternate with $\mathrm{MnO}_{6}$ octahedra along $c$, indicating the BM phase (Fig. 1C). This transformation is hysteretic and non-volatile, with LSMO remaining in the BM phase even when the external bias is removed (fig. S4) (35).

The multiple-step transformations (Fig. 1D) from $\mathrm{MnO}_{6}$ octahedra (virgin state) toward square pyramids plus octahedra $(2 \mathrm{~V})$, to alternating octahedra and tetrahedra (4 V) also correlate to the variation of the pseudo-cubic lattice parameter along the electric-field direction (called $c^{\prime}$ ). We determined the $c$ ' values at various bias voltages for the first 20 monolayers in LSMO starting from the $\mathrm{Nb}$ :STO interface (Fig. $1 \mathrm{E})$. In the virgin state, we measured $c^{\prime}$ to be $384( \pm 5) \mathrm{pm}$ with $\ddot{\mathrm{V}}_{\mathrm{o}}$ disorder induced expansion in some planes (35). At 2 $\mathrm{V}, c$ ' oscillates with values between 335 and $425 \mathrm{pm}$, without resulting in any particular superstructure. At $4 \mathrm{~V}$, except for the first few monolayers, $c$ ' alternates between 375 and 445 pm, doubling the lattice periodicity. Energy dispersive spectroscopy (35) reveals a clear gradient of oxygen concentration in the bottom electrode, compared to the virgin state, even at a low bias of $1.5 \mathrm{~V}$, with more $\ddot{\mathrm{V}}_{\mathrm{o}}$ occurring closer to the $\mathrm{Nb}$ :STO interface (Fig. 1, F and G).

Importantly, the BM phase can be reoxygenated when negative voltages are applied to the top electrode. We show this takes place for biases as low as -1 V (fig. S5A). The hysteretic BM phase clearly begins to reoxygenate in iDPC-STEM images of the same field view at $0 \mathrm{~V}$ and $-1.3 \mathrm{~V}$ (Fig. 2, A and $\mathrm{B})$, with the appearance of extra oxygen columns at $-1.3 \mathrm{~V}$ in the Mn-O planes that were oxygen deficient at $0 \mathrm{~V}$ (fig. S5A). Upon ramping the bias to $-3 \mathrm{~V}$, the entire layer converts to the BM-precursor phase (Fig. 2C) and was retained also when the bias is removed (Fig. 2D), as we confirmed by the corresponding 'disorderly' c'variation (fig. S5B).

In order to address the timescales of the processes associated with de- and re-oxygenation of LSMO layers, we followed the dynamics through HAADF-STEM image acquisition, after poling at $-4 \mathrm{~V}$ (transforming LSMO completely back to the starting perovskite phase). In the HAADF-STEM image evolution that occurs within 2 min of one region having a bias 
increase to $3 \mathrm{~V}$ (Fig. 2E), the initial perovskite phase changes to the BM-precursor phase in $60 \mathrm{~s}$, and then to the BM phase within $120 \mathrm{~s}$. These changes were indicated by the variations we observed in the $c^{\prime}$ parameter. By applying $-3 \mathrm{~V}$, we observed a complete transformation from a BM phase back to perovskite phase within $90 \mathrm{~s}$ in the region (Fig. 2F; FFT in fig. $\mathrm{S} 5 \mathrm{C})$. We then followed the same region for $10 \mathrm{~s}$ using a faster HAADF-STEM image series (1.2 s/frame). From the $c$ ' parameter variations, we concluded that the BM-precursor phase transformed to the perovskite phase (Fig. $2 \mathrm{~F}$, center panel). Thus, while the complete transformation from perovskite to BM and back takes about a couple of minutes at 3 and $-3 \mathrm{~V}$, $\ddot{\mathrm{V}}_{\mathrm{o}}$ migration and partial phase-transitions already started occurring in time scales of seconds at these voltages. At $2 \mathrm{~V}$, however, the partial transition to the BM-precursor phase itself takes 3-4 hours. The change in kinetics with voltage is consistent with the ultra non-linear "voltage-time dilemma" typically observed in oxide resistive memories (42). Thus, we expect that at higher voltages these mechanisms will occur at very short time scales.

We also monitored the structural evolution in the HZO (6 $\mathrm{nm}$ ) layer under the application of bias. From the multislice iDPC-STEM image simulations for HZO [see also (43)] in the r-phase $(R 3 m)$ with [111] out-of-plane (Fig. 3A, inset), we recognized the (001) planes (at $\sim 55^{\circ}$ with respect to the [111] direction) by cationic (Hf/Zr) columns surrounded by two oxygen columns on either side of them. In the virgin state, our experimental images perfectly match the r-phase simulations. We followed the evolution of a supercell (Fig. 3A) in this grain upon application of bias along the out of plane [111] direction. We show how the displacement of $\ddot{\mathrm{V}}_{0}$ for this supercell occurs (35) (fig. S6A) with respect to the $0 \mathrm{~V}$ configuration (Fig. 3B). While $\ddot{V}_{o}$ migrate toward the bottom electrode with increasing bias (Fig. 3B), they also gather some in-plane displacement (Fig. 3B, inset).

At $4 \mathrm{~V}$, the same grain transforms into a combination of multiple grains (Fig. 3C). Upon inspecting various regions in the film, we found that the majority of the grains have changed their structure from r-phase toward the more thermodynamically stable orthorhombic (o-) and monoclinic (m) phases (Fig. 3D and fig. S6C). The o-phase is commonly observed in ferroelectric HZO layers grown by various methods (5), while the r-phase is only observed under specific growth procedures and conditions (6). Our observations on HZO point to the r-phase being stabilized under slight oxygen deficient conditions. Replenishment of oxygen in the HZO layer under bias (originating from the bottom LSMO layer), transforms it into more stoichiometric m- or o-phases. The $\ddot{V}_{o}$ in the HZO layer, and thus the r-phase, is restored (by reverse migration) upon applying a bias of $-3 \mathrm{~V}$, as can be seen from the perfect match of the experimental iDPC-STEM of two representative domains $\left(180^{\circ}\right.$ rotated from each other) (Fig. 3E), with the multislice image simulations (Fig. 3A, inset).The $\mathrm{m} / \mathrm{o}$-phases can be reversibly obtained again in subsequent cycles of positive bias (fig. S6D).

To elucidate the effects of device cycling with sub-millisecond pulses (35), we present results on tunnel junction $\mathrm{Co} / \mathrm{HZO}(2 \mathrm{~nm}) / \mathrm{LSMO} / / \mathrm{STO}$ devices switched between lowresistance state (LRS) and high-resistance state (HRS) at large voltages of $\pm 6 \mathrm{~V}$. The devices showed an increasing TER (35) from $100 \%$ (stage A) to $10^{6 \%}$ (stage B) upon cycling $~ 100$ times $(23,44)$. An iDPC-STEM image from a selected region in the LSMO (bottom electrode) layer in the LRS (stage A) (Fig. 4A) shows a clear perovskite structure. In the HRS (stage B), however (Fig. 4B), Co inhomogeneously oxidizes exhibiting co-existing Co rich and $\mathrm{CoO}_{\mathrm{x}}$ rich regions as revealed from the EDS analysis (fig. S7). This leaves oxygen deficient LSMO in the BM precursor-phase. Thus, progression from stage A to stage $\mathrm{B}$ results in gradual increase of $\mathrm{CoO}_{\mathrm{x}}$ regions, an accumulated effect of oxygen voltammetry, which is reflected electrically in diverging resistance values.

To disentangle the short-term field effects on our devices from accumulated effects (35), we report ex situ structureproperty correlation results on ferroelectric capacitor stacks of LSMO (or TiN)/HZO (7 nm)/LSMO//STO cycled at $1 \mathrm{kHz}$ for $<10$ times at $5.5 \mathrm{~V}$. The intrinsic $\mathrm{P}_{r}$ of our virgin devices in r-phase obtained from atomic displacements (35) (fig. S5B) is $<9 \mu \mathrm{C} / \mathrm{cm}^{2}$, which is very small compared to the $35 \mu \mathrm{C} / \mathrm{cm}^{2}$ that were measured from P-V loops at room temperature (7). This discrepancy is already an indication that most of the switching charge is intertwined with extrinsic factors. From the P-V hysteresis loops at various temperatures (Fig. 4C), we observed that, contrary to what it is expected in classical ferroelectrics, the $\mathrm{P}_{r}$ increases with increasing temperature in the range from $150 \mathrm{~K}$ to $300 \mathrm{~K}(22)$. This observation is in line with the polarization switching being correlated to the thermally activated oxygen migration mechanism. These devices were prepared in a "down polarized" configuration $(5.5 \mathrm{~V}, 1$ $\mathrm{kHz}$ ) and imaged. The iDPC-STEM images clearly reveal the oxygen deficient BM-precursor phase in the bottom LSMO layer close to the HZO interface (Fig. 4D) as also confirmed by corresponding disorderly oscillations in the $c$ ' parameter (Fig. 4D, inset). Close to the STO interface, we found exaggerated oxygen octahedral tilts with $\mathrm{Mn}-\mathrm{O}-\mathrm{Mn}$ bond angles < $146^{\circ}$ (marked in fig. S8A), which is the same interfacial feature observed during in situ DC testing at $2 \mathrm{~V}$ (fig. S2B). These observations also suggest that such extreme tilts initiate the transformation from perovskite to BM-precursor phase. These incipient oxygen-migration induced topotactic structural transitions result from the discharge of $\ddot{V}_{0}$ in the electrodes. This thermally activated process leads to a built-in field at lower temperature, which decreases as the temperature rises (fig. S8, B and C). The HZO layer, however, remains in an r-phase (Fig. 4E), revealing that it just acts as a conduit 
of oxygen between the source and sink (both reactive) electrodes in the short-term.

We conducted comparative operando synchrotron nanobeam diffraction on $\mathrm{Au} / \mathrm{HZO} / \mathrm{LSMO}$ and LSMO/HZO/LSMO capacitors in order to answer the question whether oxygen voltammetry can still occur if HZO is interfaced with a nonreactive electrode in the short-term (35) (fig. S9). Both devices showed ferroelectric switching peaks (Fig. 4E and fig. S10) and we cycled them at $100 \mathrm{~Hz}$ in a modest voltage range between -3.5 and $3.5 \mathrm{~V}$ for tens of thousands of cycles (fig. S10C). In LSMO/HZO/LSMO devices the virgin state and cycled state do not show any substantial differences of the HZO lattice parameter, indicating the persistence of the r-phase during cycling (fig. S10D). However, in the case of $\mathrm{Au} / \mathrm{HZO} / \mathrm{LSMO}$, we see evidence that a monoclinic phase (with a Bragg peak at $2 \theta=24.9^{\circ}$ ) appears after cycling, and coexists with the r-phase (fig. S10, E and F). This reveals that the HZO layer itself is forced to act as source/sink of oxygen vacancies in the short-term (35), when $\mathrm{Au}$ is used as a top electrode, resulting in reversible structural phase transitions between $r$ and $m$ phases. Further DC biasing, results in increase of m-phase fraction increasing positive bias, in agreement with the long-term in situ TEM observations (fig. S11).

Using the model system of epitaxial HZO/LSMO/STO, we demonstrated that oxygen voltammetry and ferroelectric switching are intertwined (Fig. 4F) (35).With reactive top electrodes such as TiN (45), Co, and LSMO, the HZO layer acts as a mere conduit for reversible oxygen migration between the electrodes, with incipient topotactic transformations taking place in them. With a noble top electrode (Au), HZO instead itself acts as a sink/source of $\ddot{\mathrm{V}}_{\mathrm{o}}$. In the longer-term, redox-based phase-transition effects that follow oxygen migration in both HZO and LSMO become more dramatic. We clearly show that oxygen voltammetry is intertwined with ferroelectric switching of HZO. While we remain agnostic about the nature of this coupling, recent predictions of electrochemical origin of ferroelectricity in hafnia-based compounds (29), as well as demonstrations of giant electrostriction in the sister compound $\mathrm{Gd}: \mathrm{CeO}_{2},(46)$ can provide important clues.

In the context of tunnel junction devices, perovskite manganites (47) are routinely used as a back electrodes (24-27, 48). Their crucial role as oxygen conducting memristive layers (41) actively participating in charge transport is being recognized (35), with recent seminal demonstrations in ferroelectric tunnel junctions (28). Thus, our results have deep implications not just to hafnia-based ferroelectrics, but also to the wider field of oxide electronics.

\section{REFERENCES AND NOTES}

1. T. S. Böscke, J. Müller, D. Bräuhaus, U. Schröder, U. Böttger, Ferroelectricity in hafnium oxide thin films. Appl. Phys. Lett. 99, 102903 (2011). doi:10.1063/1.3634052
2. A. Chernikova, M. Kozodaev, A. Markeev, D. Negrov, M. Spiridonov, S. Zarubin, O. Bak, P. Buragohain, H. Lu, E. Suvorova, A. Gruverman, A. Zenkevich, Ultrathin $\mathrm{Hf}_{0.5} \mathrm{Zr}_{0.5} \mathrm{O}_{2}$ Ferroelectric Films on Si. ACS Appl. Mater. Interfaces 8, 7232-7237 (2016). doi:10.1021/acsami.5b11653 Medline

3. X. Tian, S. Shibayama, T. Nishimura, T. Yajima, S. Migita, A. Toriumi, Evolution of ferroelectric $\mathrm{HfO}_{2}$ in ultrathin region down to $3 \mathrm{~nm}$. Appl. Phys. Lett. 112, 102902 (2018). doi:10.1063/1.5017094

4. S. S. Cheema, D. Kwon, N. Shanker, R. Dos Reis, S.-L. Hsu, J. Xiao, H. Zhang, R. Wagner, A. Datar, M. R. McCarter, C. R. Serrao, A. K. Yadav, G. Karbasian, C.-H. Hsu, A. J. Tan, L.-C. Wang, V. Thakare, X. Zhang, A. Mehta, E. Karapetrova, R. V. Chopdekar, P. Shafer, E. Arenholz, C. Hu, R. Proksch, R. Ramesh, J. Ciston, S. Salahuddin, Enhanced ferroelectricity in ultrathin films grown directly on silicon. Nature 580, 478-482 (2020). doi:10.1038/s41586-020-2208-x Medline

5. U. Schröder, C. S. Hwang, H. Funakubo, Eds., Ferroelectricity in Doped Hafnium Oxide (Woodhead Publishing, ed. 1, 2019).

6. P. Nukala, Y. Wei, V. de Haas, Q. Guo, J. Antoja-Lleonart, B. Noheda, Guidelines for the stabilization of a polar rhombohedral phase in epitaxial $\mathrm{Hf}_{0.5} \mathrm{Zr}_{0.5} \mathrm{O}_{2}$ thin films. Ferroelectrics 569, 148-163 (2020). doi:10.1080/00150193.2020.1791658

7. Y. Wei, P. Nukala, M. Salverda, S. Matzen, H. J. Zhao, J. Momand, A. S. Everhardt, G. Agnus, G. R. Blake, P. Lecoeur, B. J. Kooi, J. Íniguez, B. Dkhil, B. Noheda, A rhombohedral ferroelectric phase in epitaxially strained $\mathrm{Hf}_{0.5} \mathrm{Zr}_{0.5} \mathrm{O}_{2}$ thin films. Nat. Mater. 17, 1095-1100 (2018). doi:10.1038/s41563-018-0196-0 Medline

8. R. Materlik, C. Kunneth, A. Kersch, The origin of ferroelectricity in $\mathrm{Hf}_{1-x} \mathrm{Zr}_{x} \mathrm{O}_{2}$ : A computational investigation and a surface energy model. J. Appl. Phys. 117, 134109 (2015). doi:10.1063/1.4916707

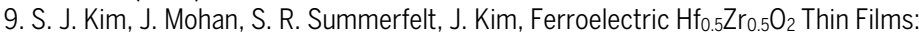
A Review of Recent Advances. JOM 71, 246-255 (2019). doi:10.1007/s11837-018$\underline{3140-5}$

10. T. Mimura, T. Shimizu, H. Funakubo, Ferroelectricity in $\mathrm{YO}_{1.5}-\mathrm{HfO}_{2}$ films around 1 $\mu \mathrm{m}$ in thickness. Appl. Phys. Lett. 115, 032901 (2019). doi:10.1063/1.5097880

11. X. Xu, F.-T. Huang, Y. Qi, S. Singh, K. M. Rabe, D. Obeysekera, J. Yang, M.-W. Chu, S.-W. Cheong, Kinetically stabilized ferroelectricity in bulk single-crystalline HfO :Y. Nat. Mater. 10.1038/s41563-020-00897-x (2021). doi:10.1038/s41563020-00897-x Medline

12. Y. Zhou, Y. K. Zhang, Q. Yang, J. Jiang, P. Fan, M. Liao, Y. C. Zhou, The effects of oxygen vacancies on ferroelectric phase transition of $\mathrm{HfO}_{2}$-based thin film from first-principle. Comput. Mater. Sci. 167, 143-150 (2019). doi:10.1016/i.commatsci.2019.05.041

13. H. Mulaosmanovic, J. Ocker, S. Müller, U. Schroeder, J. Müller, P. Polakowski, S. Flachowsky, R. van Bentum, T. Mikolajick, S. Slesazeck, Switching kinetics in nanoscale hafnium oxide based ferroelectric field-effect transistors. ACS Appl. Mater. Interfaces 9, 3792-3798 (2017). doi:10.1021/acsami.6b13866 Medline

14. M. Pešić, C. Künneth, M. Hoffmann, H. Mulaosmanovic, S. Müller, E. T. Breyer, U. Schroeder, A. Kersch, T. Mikolajick, S. Slesazeck, A computational study of hafniabased ferroelectric memories: From ab initio via physical modeling to circuit models of ferroelectric device. J. Comput. Electron. 16, 1236-1256 (2017). doi:10.1007/s10825-017-1053-0

15. N. Gong, X. Sun, H. Jiang, K. S. Chang-Liao, Q. Xia, T. P. Ma, Nucleation limited switching (NLS) model for $\mathrm{HfO}_{2}$-based metal-ferroelectric-metal (MFM) capacitors: Switching kinetics and retention characteristics. Appl. Phys. Lett. 112, 262903 (2018). doi:10.1063/1.5010207

16. P. Buragohain, C. Richter, T. Schenk, H. Lu, T. Mikolajick, U. Schroeder, A. Gruverman, Nanoscopic studies of domain structure dynamics in ferroelectric La: $\mathrm{HfO}_{2}$ capacitors. Appl. Phys. Lett. 112, 222901 (2018). doi:10.1063/1.5030562

17. S. Migita, H. Ota, H. Yamada, K. Shibuya, A. Sawa, A. Toriumi, Polarization switching behavior of $\mathrm{Hf}-\mathrm{Zr}-\mathrm{O}$ ferroelectric ultrathin films studied through coercive field characteristics. Jpn. J. Appl. Phys. 57, 04FB01 (2018). doi:10.7567/JJAP.57.04FB01

18. Y. H. Lee, S. D. Hyun, H. J. Kim, J. S. Kim, C. Yoo, T. Moon, K. D. Kim, H. W. Park, Y. B. Lee, B. S. Kim, J. Roh, M. H. Park, C. S. Hwang, Nucleation-Limited Ferroelectric Orthorhombic Phase Formation in $\mathrm{Hf}_{0.5} \mathrm{Zr}_{0.5} \mathrm{O}_{2}$ Thin Films. Adv. Electron. Mater. 5, 180436 (2019). doi:10.1002/aelm.201800436

19. T. Schenk, M. Hoffmann, J. Ocker, M. Pešić, T. Mikolajick, U. Schroeder, Complex internal bias fields in ferroelectric hafnium oxide. ACS Appl. Mater. Interfaces 7 , 20224-20233 (2015). doi:10.1021/acsami.5b05773 Medline 
20. H.-J. Lee, M. Lee, K. Lee, J. Jo, H. Yang, Y. Kim, S. C. Chae, U. Waghmare, J. H. Lee, Scale-free ferroelectricity induced by flat phonon bands in $\mathrm{HfO}_{2}$. Science 369 , 1343-1347 (2020). doi:10.1126/science. aba0067 Medline

21. C. Li, Y. Yao, X. Shen, Y. Wang, J. Li, C. Gu, R. Yu, Q. Liu, M. Liu, Dynamic observation of oxygen vacancies in hafnia layer by in situ transmission electron microscopy. Nano Res. 8, 3571-3579 (2015). doi:10.1007/s12274-015-0857-0

22. S. U. Sharath, S. Vogel, L. Molina-Luna, E. Hildebrandt, C. Wenger, J. Kurian, M. Duerrschnabel, T. Niermann, G. Niu, P. Calka, M. Lehmann, H. J. Kleebe, T. Schroeder, L. Alff, Control of Switching Modes and Conductance Quantization in Oxygen Engineered $\mathrm{HfO}_{\mathrm{x}}$ based Memristive Devices. Adv. Funct. Mater. 27, 1700432 (2017). doi:10.1002/adfm.201700432

23. Y. Wei, S. Matzen, C. P. Quinteros, T. Maroutian, G. Agnus, P. Lecoeur, B. Noheda, Magneto-ionic control of spin polarization in multiferroic tunnel junctions. $n p j$ Quantum Mater. 4, 62 (2019). doi:10.1038/s41535-019-0201-0

24. M. C. Sulzbach, S. Estandía, X. Long, J. Lyu, N. Dix, J. Gàzquez, M. F. Chisholm, F. Sánchez, I. Fina, J. Fontcuberta, Unraveling Ferroelectric Polarization and Ionic

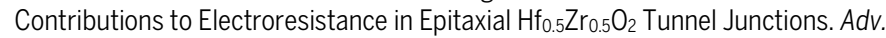
Electron. Mater. 6, 1900852 (2020). doi:10.1002/aelm.201900852

25. M. Hambe, A. Petraru, N. A. Pertsev, P. Munroe, V. Nagarajan, H. Kohlstedt, Crossing an interface: Ferroelectric control of tunnel currents in magnetic complex oxide heterostructures. Adv. Funct. Mater. 20, 2436-2441 (2010). doi:10.1002/adfm.201000265

26. D. Pantel, S. Goetze, D. Hesse, M. Alexe, Room-temperature ferroelectric resistive switching in ultrathin $\mathrm{Pb}\left(\mathrm{Zr}_{0.2} \mathrm{Ti}_{0.8}\right) \mathrm{O}_{3}$ films. ACS Nano 5, 6032-6038 (2011). doi:10.1021/nn2018528 Medline

27. S. Boyn, J. Grollier, G. Lecerf, B. Xu, N. Locatelli, S. Fusil, S. Girod, C. Carrétéro, K. Garcia, S. Xavier, J. Tomas, L. Bellaiche, M. Bibes, A. Barthélémy, S. Saïghi, V. Garcia, Learning through ferroelectric domain dynamics in solid-state synapses. Nat. Commun. 8, 14736 (2017). doi:10.1038/ncomms14736 Medline

28. V. Rouco, R. E. Hage, A. Sander, J. Grandal, K. Seurre, X. Palermo, J. Briatico, S. Collin, J. Trastoy, K. Bouzehouane, A. I. Buzdin, G. Singh, N. Bergeal, C. FeuilletPalma, J. Lesueur, C. Leon, M. Varela, J. Santamaría, J. E. Villegas, Quasiparticle tunnel electroresistance in superconducting junctions. Nat. Commun. 11, 658 (2020). doi:10.1038/s41467-020-14379-w Medline

29. V. Mikheev, A. Chouprik, Y. Lebedinskii, S. Zarubin, Y. Matveyev, E. Kondratyuk, M. G. Kozodaev, A. M. Markeev, A. Zenkevich, D. Negrov, Ferroelectric Second-Order Memristor. ACS Appl. Mater. Interfaces 11, 32108-32114 (2019). doi:10.1021/acsami.9b08189 Medline

30. M. D. Glinchuk, A. N. Morozovska, A. Lukowiak, W. Stręk, M. V. Silibin, D. V. Karpinsky, Y. Kim, S. V. Kalinin, Possible electrochemical origin of ferroelectricity in $\mathrm{HfO}_{2}$ thin films. J. Alloys Compd. 830, 153628 (2020). doi:10.1016/i.jallcom.2019.153628

31. M. Qian, I. Fina, M. C. Sulzbach, F. Sánchez, J. Fontcuberta, Synergetic Electronic and Ionic Contributions to Electroresistance in Ferroelectric Capacitors. Adv. Electron. Mater. 5, 1800646 (2019). doi:10.1002/aelm.201800646

32. C. Ferreyra, M. Rengifo, M. J. Sánchez, A. S. Everhardt, B. Noheda, D. Rubi, Key Role of Oxygen-Vacancy Electromigration in the Memristive Response of Ferroelectric Devices. Phys. Rev. Appl. 14, 044045 (2020). doi:10.1103/PhysRevApplied.14.044045

33. M. Pešić, F. P. G. Fengler, L. Larcher, A. Padovani, T. Schenk, E. D. Grimley, X. Sang, J. M. LeBeau, S. Slesazeck, U. Schroeder, T. Mikolajick, Physical Mechanisms behind the Field-Cycling Behavior of $\mathrm{HfO}_{2}$-Based Ferroelectric Capacitors. Adv. Funct. Mater. 26, 4601-4612 (2016). doi:10.1002/adfm.201600590

34. P. Gao, C. T. Nelson, J. R. Jokisaari, S. H. Baek, C. W. Bark, Y. Zhang, E. Wang, D. G. Schlom, C. B. Eom, X. Pan, Revealing the role of defects in ferroelectric switching with atomic resolution. Nat. Commun. 2, 591 (2011). doi: $10.1038 /$ ncomms 1600 Medline

35. Materials and methods are available as supplementary materials online.

36. S. de Graaf, J. Momand, C. Mitterbauer, S. Lazar, B. J. Kooi, Resolving hydrogen atoms at metal-metal hydride interfaces. Sci. Adv. 6, eaay4312 (2020). doi:10.1126/sciadv.aay4312 Medline

37. G. Sanchez-Santolino, M. Cabero, M. Varela, J. Garcia-Barriocanal, C. Leon, S. J. Pennycook, J. Santamaria, Oxygen octahedral distortions in $\mathrm{LaMO}_{3} / \mathrm{SrTiO}_{3}$ superlattices. Microsc. Microanal. 20, 825-831 (2014). doi:10.1017/S1431927614000750 Medline
38. S. Stølen, E. Bakken, C. E. Mohn, Oxygen-deficient perovskites: Linking structure, energetics and ion transport. Phys. Chem. Chem. Phys. 8, 429-447 (2006). doi:10.1039/B512271E Medline

39. P. Nukala, M. Ahmadi, J. Antoja-Lleonart, S. de Graaf, Y. Wei, H. W. Zandbergen, B. J. Kooi, B. Noheda, In situ heating studies on temperature-induced phase transitions in epitaxial $\mathrm{Hf}_{0.5} \mathrm{Zr}_{0.5} \mathrm{O}_{2} / \mathrm{La}_{0.67} \mathrm{Sr}_{0.33} \mathrm{MnO}_{3}$ heterostructures. Appl. Phys. Lett. 118, 062901 (2021). doi:10.1063/5.0035714

40. L. Cao, O. Petracic, P. Zakalek, A. Weber, U. Rücker, J. Schubert, A. Koutsioubas, S. Mattauch, T. Brückel, Reversible Control of Physical Properties via an OxygenVacancy-Driven Topotactic Transition in Epitaxial La0.7 $\mathrm{Sr}_{0.3} \mathrm{MnO}_{3-\delta}$ Thin Films. Adv. Mater. 31, 1806183 (2019). doi:10.1002/adma.201806183

41. L. Yao, S. Inkinen, S. van Dijken, Direct observation of oxygen vacancy-driven structural and resistive phase transitions in $\mathrm{La}_{2 / 3} \mathrm{Sr}_{1 / 3} \mathrm{MnO}_{3}$. Nat. Commun. 8, 14544 (2017). doi:10.1038/ncomms 14544 Medline

42. C. Baeumer, C. Schmitz, A. H. H. Ramadan, H. Du, K. Skaja, V. Feyer, P. Müller, B. Arndt, C. L. Jia, J. Mayer, R. A. De Souza, C. Michael Schneider, R. Waser, R. Dittmann, Spectromicroscopic insights for rational design of redox-based memristive devices. Nat. Commun. 6, 8610 (2015). doi:10.1038/ncomms9610 Medline

43. L. Bégon-Lours, M. Mulder, P. Nukala, S. De Graaf, Y. A. Birkhölzer, B. Kooi, B. Noheda, G. Koster, G. Rijnders, Stabilization of phase-pure rhombohedral $\mathrm{HfZrO}_{4}$ in pulsed laser deposited thin films. Phys. Rev. Mater. 4, 043401 (2020). doi:10.1103/PhysRevMaterials.4.043401

44. Y. Wei, S. Matzen, T. Maroutian, G. Agnus, M. Salverda, P. Nukala, Q. Chen, J. Ye, P. Lecoeur, B. Noheda, Magnetic Tunnel Junctions Based on Ferroelectric $\mathrm{Hf}_{0.5} \mathrm{Zr}_{0.5} \mathrm{O}_{2}$ Tunnel Barriers. Phys. Rev. Appl. 12, 031001 (2019). doi:10.1103/PhysRevApplied.12.031001

45. W. Hamouda, C. Lubin, S. Ueda, Y. Yamashita, O. Renault, F. Mehmood, T. Mikolajick, U. Schroeder, R. Negrea, N. Barrett, Interface chemistry of pristine

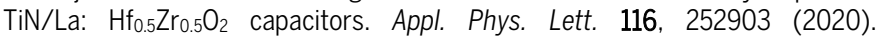
doi:10.1063/5.0012595

46. S. Santucci, S. Sanna, N. Pryds, V. Esposito, "Defective Metal Oxides-New Generation of Electrostrictor Materials" in Proceedings of 2018 MRS Fall Meeting and Exhibition (Materials Research Society, EP01.01.02, 2016).

47. A. Herpers, C. Lenser, C. Park, F. Offi, F. Borgatti, G. Panaccione, S. Menzel, R. Waser, R. Dittmann, Spectroscopic proof of the correlation between redox-state and charge-carrier transport at the interface of resistively switching Ti/PCMO devices. Adv. Mater. 26, 2730-2735 (2014). doi:10.1002/adma.201304054 Medline

48. G. Radaelli, D. Gutiérrez, F. Sánchez, R. Bertacco, M. Stengel, J. Fontcuberta, Large room-temperature electroresistance in dual-modulated ferroelectric tunnel barriers. Adv. Mater. 27, 2602-2607 (2015). doi:10.1002/adma.201405117 Medline

49. P. Nukala, M. Ahmadi, Y. Wei, S. de Graaf, E. Stylianidis, T. Chakrabortty, S. Matzen, H. W. Zandbergen, A. Björling, D. Mannix, D. Carbone, B. Kooi, B. Noheda, Dataset: Reversible oxygen migration and phase transitions in hafnia-based ferroelectric devices, version 1.0, Dataverse NL (2021); https://doi.org/10.34894/EIOXPI.

50. U. Johansson, U. Voigt, A. Mikkelsen, NanoMAX: A hard x-ray beamline at MAX IV Proc. SPIE 8851, 88510L (2013). doi:10.1117/12.2026609

51. A. Björling, S. Kalbfleisch, M. Kahnt, S. Sala, K. Parfeniukas, U. Vogt, D. Carbone, U. Johansson, Ptychographic characterization of a coherent nanofocused X-ray beam. Opt. Express 28, 5069-5076 (2020). doi:10.1364/0E.386068 Medline

52. Z. Horita, T. Sano, M. Nemoto, Energydispersive $x$-ray microanalysis in the analytical electron microscope. ISIJ Int. 29, 179-190 (1989). doi:10.2355/isiijnternational.29.179

53. F. P. G. Fengler, M. Pešić, S. Starschich, T. Schneller, C. Künneth, U. Böttger, H. Mulaosmanovic, T. Schenk, M. H. Park, R. Nigon, P. Muralt, T. Mikolajick, U. Schroeder, Domain Pinning: Comparison of Hafnia and PZT Based Ferroelectrics. Adv. Electron. Mater. 3, 1600505 (2017). doi:10.1002/aelm.201600505

\section{ACKNOWLEDGMENTS}

Funding: PN would like to acknowledge funding from European Union's Horizon 2020 research and innovation program under Marie Sklodowska-Curie grant agreement \#794954 (nickname: FERHAZ). MA, YW, BJK and BN acknowledge 
the financial support of the CogniGron research center and the Ubbo Emmius Funds (Univ. of Groningen). YW and SM acknowledge a China Scholarship Council grant and a Van Gogh travel grant. We acknowledge MAX IV Laboratory for time on Beamline NanoMAX under Proposal 20190954. Research conducted at MAX IV, a Swedish national user facility, is supported by the Swedish Research council under contract 2018-07152, the Swedish Governmental Agency for Innovation Systems under contract 2018-04969, and Formas under contract 2019-02496. SM acknowledges the French RENATECH network that supported the cleanroom activity. PN acknowledges all the discussions with Pratyush Buragohain from the University of Nebraska. Author contributions: PN, BN, MA and DC conceived the idea. PN synthesized the samples through PLD. MA, PN devised the in situ biasing device preparation protocol using FIB, and made the devices. PN, MA and SG set-up the electrical biasing system compatible with Themis-Z microscope. PN, MA and SG carried out the in situ biasing experiments with timely help from HWZ and BJK. YF and SM synthesized and fabricated tunnel junction and capacitor devices and did the ex situ electrical testing. PN, MA prepared the lamellae and carried out imaging experiments. PN, MA, YF, TC and $S G$ analyzed the TEM and transport data. DC, AB and DM carried out the experiments on the beamline. ES, PN, DC and AB carried out synchrotron data analysis. All the authors discussed the data. PN, BN co-wrote the manuscript, which was read, edited and approved by all the authors. Competing interests: The authors declare no competing interests. Data and materials availability: The data are available from the University of Groningen data repository (49).

\section{SUPPLEMENTARY MATERIALS}

science.sciencemag.org/cgi/content/full/science.abf3789/DC1

Materials and Methods

Supplementary Text

Figs. S1 to S11

References (50-53)

22 October 2020; accepted 2 April 2021

Published online 15 April 2021

10.1126/science.abf3789 
A

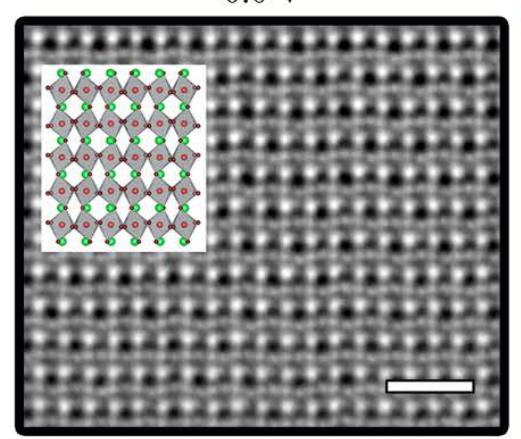

D
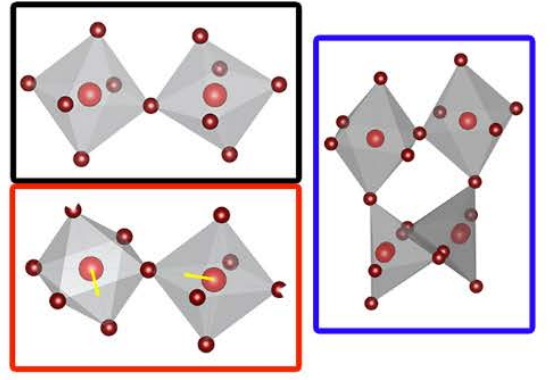

B

$2.0 \mathrm{~V}$
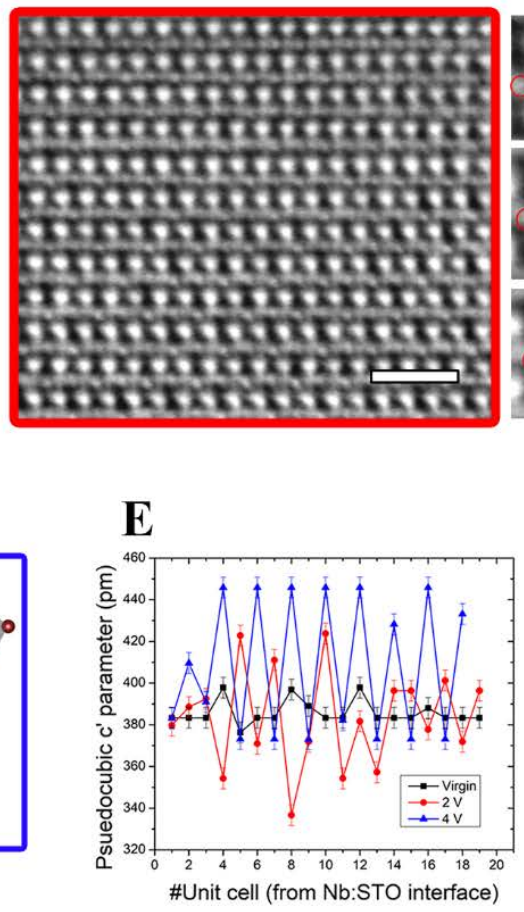

C

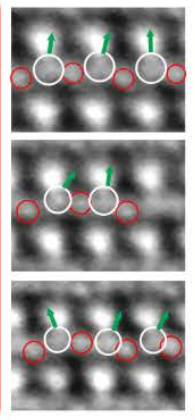

F

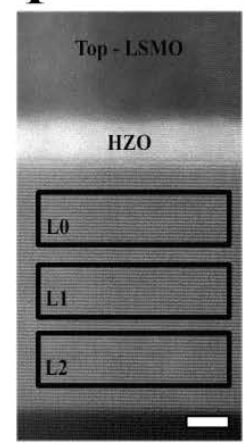

$4.0 \mathrm{~V}$
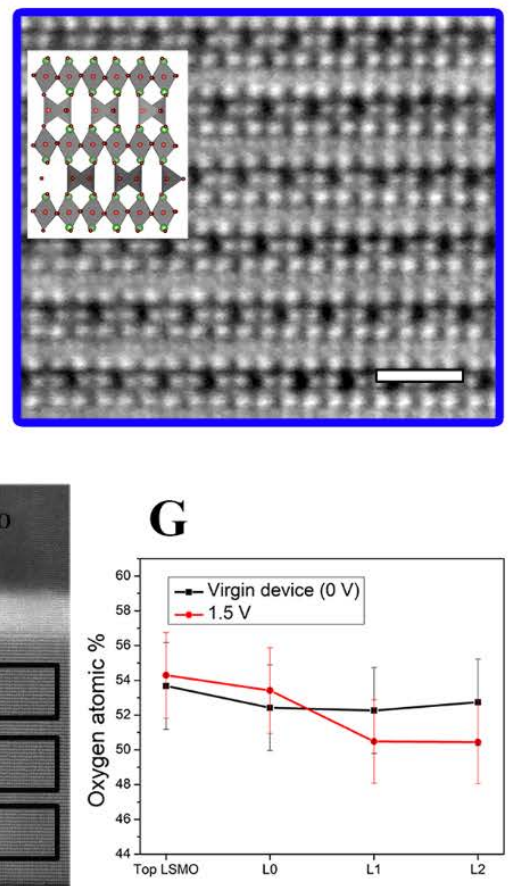

Fig. 1. Deoxygenation of bottom electrode LSMO layer with increasing positive bias. (A) iDPC-STEM image of a representative region of the bottom LSMO layer in the virgin state, viewed along the [110] zone axis, exactly matching the perovskite structure. Schematic in the inset showing $\mathrm{MnO}_{6}$ octahedra and their antiphase tilts, clearly imaged in (A). (inset) La/Sr: green, Mn: red, O:brown). (B) iDPC-STEM image at V=2 $\checkmark$. Panels on the right show various unit cells, illustrating Mn columns (circled in white) and their displacements (marked by green arrows) away from the center of an octahedron. Oxygen columns are marked in red circles. (C) iDPC-STEM at $4 \mathrm{~V}$. BM LSMO (zone axis: a, schematic in inset) denoted by alternating $\mathrm{MnO}_{4}$ tetrahedra and $\mathrm{MnO}_{6}$ octahedra along c'. (D) Schematic showing the evolution of an $\mathrm{MnO}_{6}$ octahedra in the virgin state (enclosed in black box) toward $\mathrm{MnO}_{5}$ square pyramids at $2 \mathrm{~V}$ (enclosed in red box) to alternating $\mathrm{MnO}_{4}$ tetrahedra and $\mathrm{MnO}_{6}$ octahedra at $4 \mathrm{~V}$ (enclosed in blue). (E) Plot of variation of $c^{\prime}$ (La-La distance) parameter from the STO interface in perovskite (black), BM-precursor (red) and BM phases (blue). (F) Overview image of LSMO/HZO/LSMO capacitor with regions marked where oxygen content was quantified from $\operatorname{EDS}(G)$ at $O V$ and $1.5 \mathrm{~V}$. Scale bars, $1 \mathrm{~nm}$ in $(A-C)$, and $5 \mathrm{~nm}$ in $(F)$. 


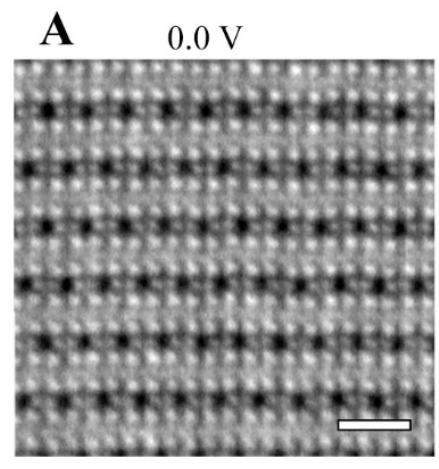

E

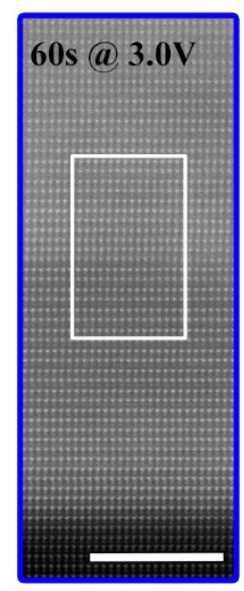

B

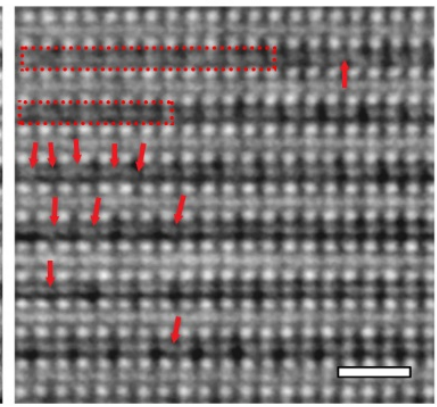

D $0.0 \mathrm{~V}$
C

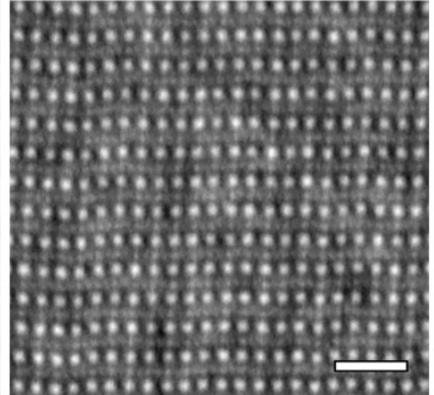

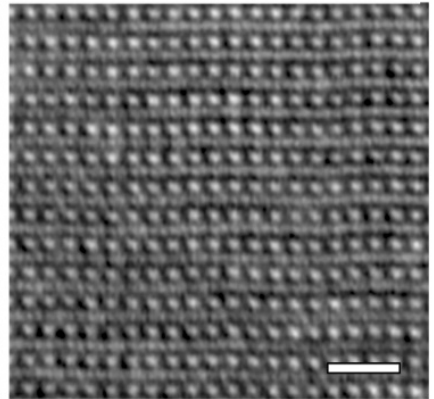

$\mathbf{F}$
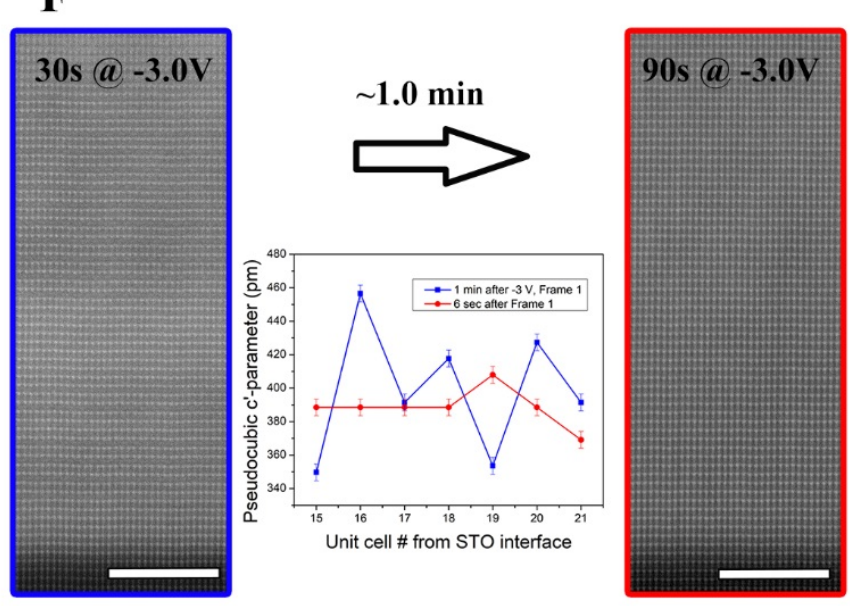

Fig. 2. Oxygenation of bottom electrode LSMO layer with increasing negative bias. iDPC-STEM images of a region in the same field of view at $O(A)$ and $-1.3 \mathrm{~V}(\mathrm{~B})$. At $-1.3 \mathrm{~V}$ oxygen columns start to appear (marked by red arrows) in positions where there were none in the BM phase at $\mathrm{O} V$. (C) BM phase transforms to BM precursor phase at $-3 \mathrm{~V}$ and is retained so at $\mathrm{O} V(\mathrm{D})$. (E) Dynamics is recorded via HAADF-STEM imaging within $120 \mathrm{~s}$ of ramping from 0 to $3 \mathrm{~V}$ from a starting perovskite phase. A BM-precursor phase is imaged at $60 \mathrm{~s}$, BM phase is imaged at $120 \mathrm{~s}$. (F) Upon changing the bias to $-3 \mathrm{~V}$, a BM phase is recorded at $30 \mathrm{~s}$, changes to perovskite phase by $90 \mathrm{~s}$ (disappearance of the superstructure spots in FFT, fig. S4). The intermediate BM-precursor phase recorded at $60 \mathrm{~s}$, converts to perovskite-like phase in about $6 \mathrm{~s}$ as can be seen by the variation in c' parameter, giving an idea about the time scales of $\ddot{V}_{\circ}$ migration. Scale bars: $1 \mathrm{~nm}$ in (A-D) and $5 \mathrm{~nm}$ in (E-F). 

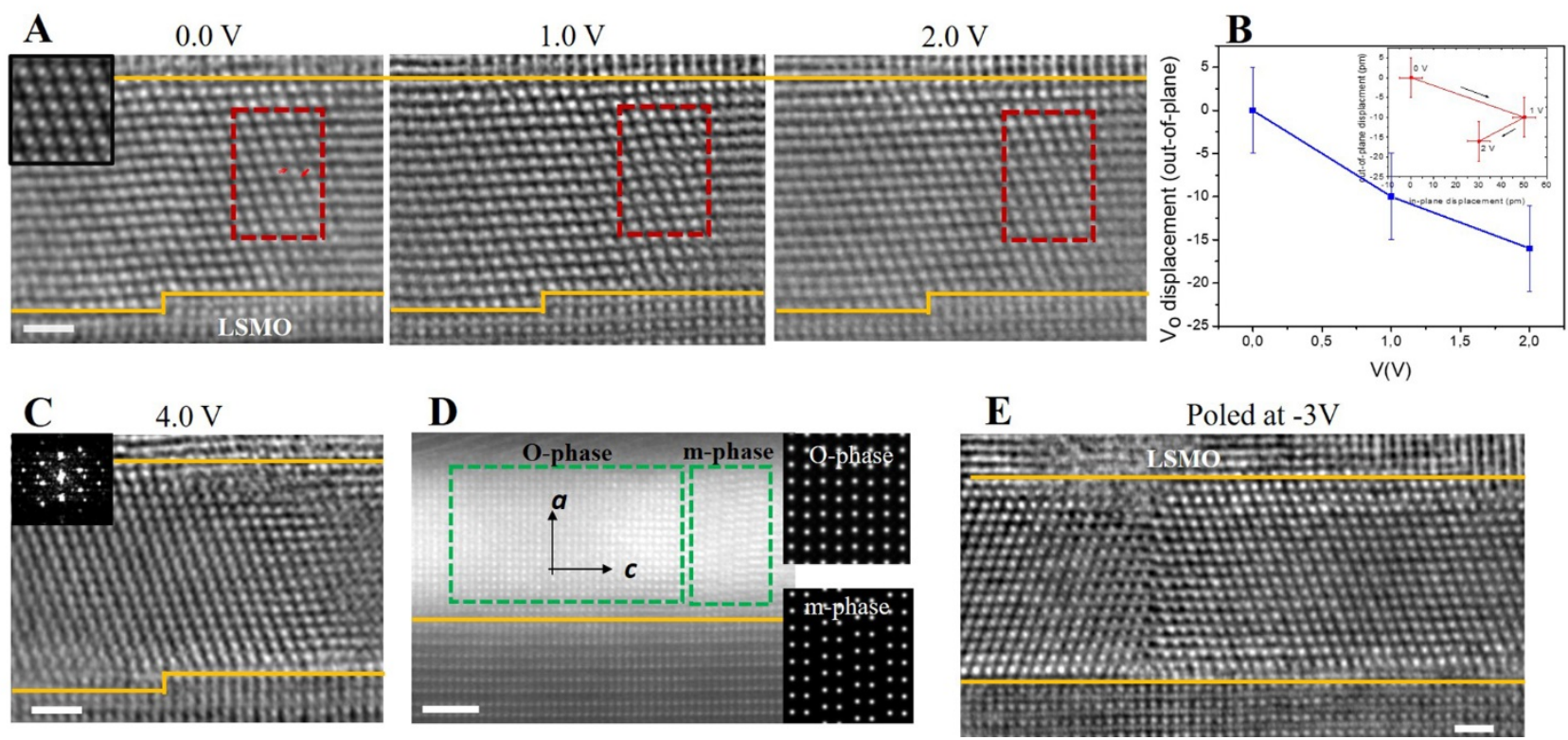

Fig. 3. Oxygenation and deoxygenation of $\mathrm{HZO}$ and associated phase transitions. (A) Evolution of an $r$-phase $\mathrm{HZO}$ grain while oxygenating under positive bias followed through iDPC-STEM image, where both cations and oxygen columns are displayed. The image of the virgin state at OV shows two red arrows pointing to two oxygen columns in the (100) planes neighboring a cationic column. Multislice iDPC-STEM image simulations of the r-phase ( $R 3 m$ symmetry) in the inset shows the good match with the observations. (B) Out-of-plane displacement of $\ddot{V}_{\circ}$ with external bias, in the marked supercell (red box) with respect to the positions in (A). Negative values indicate displacement toward bottom electrode. $\ddot{V}_{0}$ shows both in-plane and out-of-plane (toward bottom electrode) components (inset). (C) A new grain nucleates in the same region at $4 \mathrm{~V}$, giving rise to a polycrystalline nature (FFT in inset). (D) Another region in the $\mathrm{HZO}$ film back at $\mathrm{O} V$ showing o-phase and $\mathrm{m}$-phase (with multislice simulations of both in the insets). Also note the change of orientation from [111] to [100]. (E) iDPC-STEM image of domains (mutually rotated by $180^{\circ}$ about [111]) in the $r$-phase [to be compared with simulation in inset of figure $3 a$ from (36)], which is retained when poled at $-3 \mathrm{~V}$ (imaged at $O \mathrm{~V}$ ). Scale bars: $1 \mathrm{~nm}$ in (A, C, E) $2 \mathrm{~nm}$ in (D). Interfaces between HZO and top and bottom LSMO are marked in orange. In (D) only $\mathrm{HZO} /$ bottom electrode interface is shown. 
$\mathbf{A}$

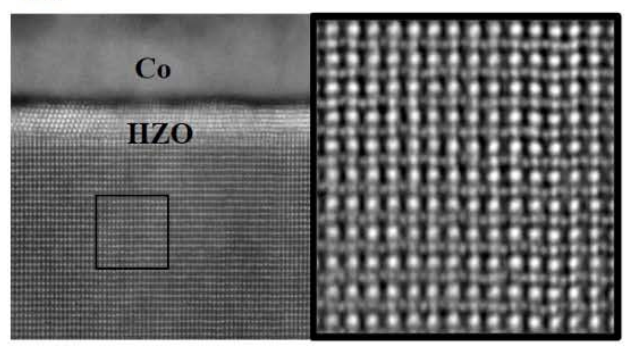

B
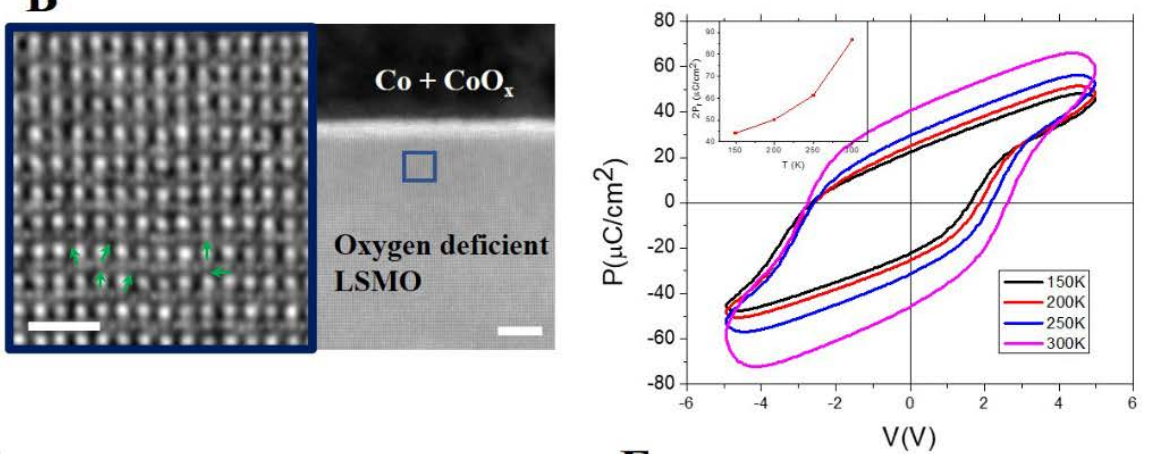

D

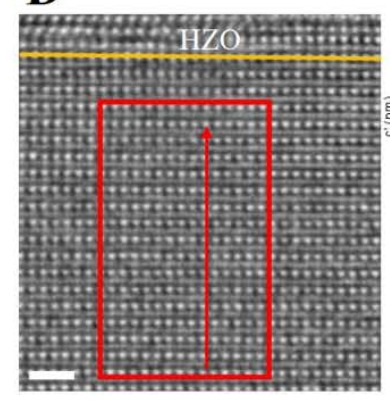

$\mathbf{E}$

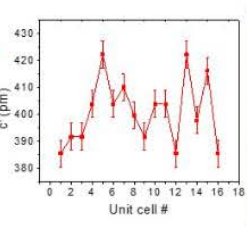

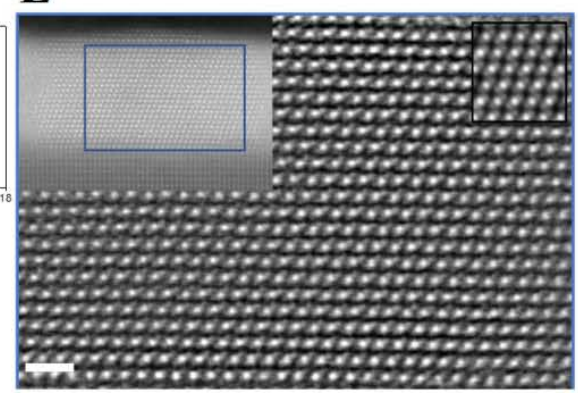
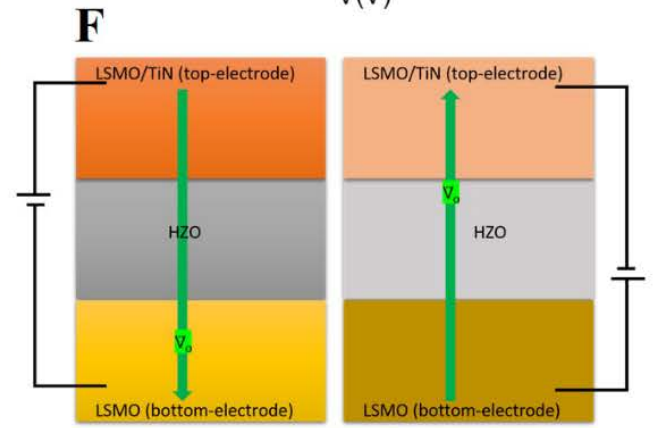

Fig. 4. Cycled tunnel junctions and ferroelectric capacitors. (A) Co/HZO (2 nm)/LSMO tunnel junction in LRS (500 $\mu \mathrm{s}, 100 \mathrm{~s}$ of cycles poled at $-6 \mathrm{~V}$ ). HAADF-STEM image on the left (scale bar: $5 \mathrm{~nm}$ ), and iDPC-STEM of a selected region in LSMO on the right (scale bar: $1 \mathrm{~nm}$ ). (B) Ex-situ imaging of tunnel junction in HRS (500 $\mu \mathrm{s}, 100 \mathrm{~s}$ of cycles, poled at $6 \mathrm{~V}$ ). (right) HAADF-STEM (and corresponding information from EDS) shows CoOx/HZO/LSMO stack (scale bar: $5 \mathrm{~nm}$ ). (left) iDPC-STEM of a region in LSMO shows BM-precursor phase (scale bar: $1 \mathrm{~nm}$ ). Selected Mn column displacements toward a square pyramidal geometry are marked by green arrows. (C) Temperature dependent P-V loops obtained from dynamic hysteresis measurements at $1 \mathrm{kHz}$ in LSMO/HZO (7 nm)/LSMO (30 $\mathrm{nm}$ ) capacitors. Inset shows the corresponding $2 \mathrm{P}_{r}$ vs T curve (see also fig. S8). (D) iDPC-STEM image of LSMO close to $\mathrm{HZO}$ interface (marked in orange) in TiN/HZO $(7 \mathrm{~nm}) / \mathrm{LSMO}$ capacitors, prepared in "down polarized" state (at $5.5 \mathrm{~V}$ ). BM-precursor phase is formed, confirmed by disorder in c' parameter (inset). (E) iDPC-STEM image of a "down" polarized HZO layer, which is structurally still in r-phase (inset left, HAADF-STEM image with the IDPC-STEM image presented for the region in the blue box; inset right, multislice simulation of $R 3 m$ phase). Scale bar in (D) and (E): $1 \mathrm{~nm}$. (F) Schematic of the polarity dependent oxygen voltammetry process in the Metal-Insulator-Metal device structure. Oxygen deficient phases in various layers are represented with lighter colors. 